\title{
Observation of the Ettingshausen effect in quantum Hall systems
}

\author{
Yosuke Komor:* and Tohru Okamotd \\ Department of Physics, University of Tokyo, Hongo 7-3-1, Bunkyo-ku, Tokyo 113-0033, Japan
}

(Dated: December 7, 2018)

\begin{abstract}
Evidence of the Ettingshausen effect in the breakdown regime of the integer quantum Hall effect has been observed in a GaAs/AlGaAs two-dimensional electron system. Resistance of micro Hall bars attached to both edges of a current channel shows remarkable asymmetric behaviors which indicate an electron temperature difference between the edges. The sign of the difference depends on the direction of the electric current and the polarity of the magnetic field. The results are consistent with the recent theory of Akera.
\end{abstract}

PACS numbers: 73.43.-f, 72.20.My, 72.20.Ht

A two-dimensional electron system (2DES) at low temperatures and in a strong magnetic field shows the quantum Hall effect (QHE), in which the longitudinal resistivity $\rho_{x x}$ vanishes and the Hall resistance $R_{\mathrm{H}}$ in a Hall bar sample is quantized as $R_{\mathrm{H}}=h / i e^{2}$ with an integer $i .1,2$ The quantized Hall resistance has been used as an international resistance standard since 1990.3 In order to carry out high precision measurements of the quantized Hall resistance, we must pass a large electric current through a Hall bar to generate a high Hall voltage across the sample. When the current exceeds a critical value, however, the QHE breaks down with a sharp increase in $\rho_{x x}$ from zero. ${ }^{4}$ In the breakdown regime, the electron temperature $T_{e}$ is expected to be much higher than the lattice temperature $T_{\mathrm{L}}$ due to the Joule heating of electrons. The hot electrons moving in the electric field carry the heat. In order to understand the mechanism of the breakdown of the QHE, it is important to know the spatial distribution of $T_{e}$ and the heat flow in a Hall bar sample. In the direction of the electric current, the variation of $T_{e}$ can be investigated by measuring the longitudinal resistance with a set of voltage probes distributed along the current channel ${ }^{5,6,7.8}$ On the other hand, it has not been studied experimentally in the direction across the current channel.

In general, the appearance of a temperature gradient or a heat flow perpendicular to both the electric current and the magnetic field is known as the Ettingshausen effect. According to the Onsager reciprocity relations, it is the cross effect of the Nernst effect, which has been observed in 2DES's. ${ }^{9,10}$ Very recently, Akera has derived hydrodynamic equations for quantum Hall systems in the regime of large energy dissipation and exhibited the spatial variation of $T_{e}$ in the direction perpendicular to the electric current ${ }^{11}$ His calculation shows that, although $T_{e}$ is approximately uniform in the middle of the current channel, it is higher around one edge and lower around the other.

In this work, we have investigated the Ettingshausen effect in a two-dimensional GaAs/AlGaAs channel using micro Hall bars attached to both edges as indicators of $T_{e}$. Asymmetric behaviors which correspond to an electron temperature difference between the edges are observed in the QHE breakdown regime. The sign of the difference is consistent with Akera's theory. The thermal relaxation length of hot electrons is also discussed.

The samples are fabricated on a $\mathrm{GaAs} / \mathrm{Al}_{0.3} \mathrm{Ga}_{0.7} \mathrm{As}$ heterostructure wafer with an electron density $N_{s}=$ $2.3 \times 10^{15} \mathrm{~m}^{-2}$ and a mobility $\mu=36 \mathrm{~m}^{2} / \mathrm{Vs}$ at $4.2 \mathrm{~K}$. As shown in Fig. 1(a), $600 \mu \mathrm{m}$ wide current electrodes are separated from the central part by $1200 \mu \mathrm{m}$ in order to avoid the effect of electron heating at the diagonally opposite corners ${ }^{12}$ A similar design was used by Kawaji et al. 13 who studied the intrinsic breakdown of the QHE and found the linear relationship between the critical current and the width of the central channel. The central part shown in Fig. 1(b) consists of the main channel whose width is $60 \mu \mathrm{m}$, four voltage probes for the measurements of $I-V$ characteristics of the main channel, and two micro Hall bars (MHBs) attached to the main channel (see Fig. 1(c)). The longitudinal resistivity in the MHBs is expected to vary with $T_{e}$ in the vicinity of each edge. All the measurements were performed at $T_{\mathrm{L}}=4.2 \mathrm{~K}$ in liquid helium.

Fig. 2 shows the longitudinal voltage drop $V_{\text {MAIN }}$ along the main channel as a function of the electric

(a)

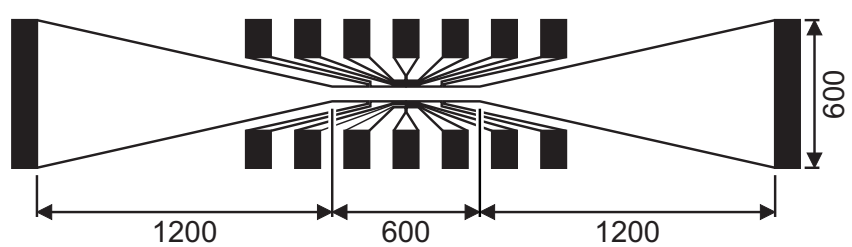

(b)

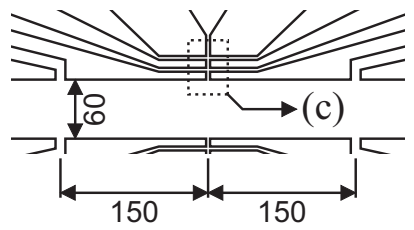

(c)

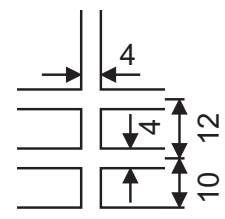

FIG. 1: Schematic view of the sample geometry. The numbers are in units of $\mu \mathrm{m}$. (a) Overall structure. (b) Structure of the central part. (c) Micro Hall bar attached to the main channel. 


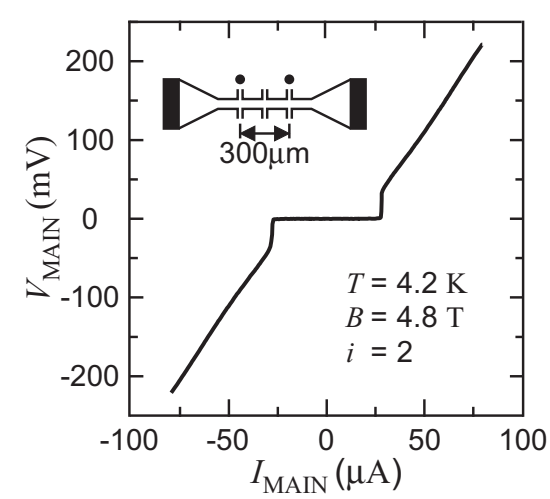

FIG. 2: $I-V$ characteristics of the main channel.

current $I_{\text {MAIN. }}$ The magnetic field $B=4.8 \mathrm{~T}$ corresponds to the center of the quantum Hall plateau with $i=2$. $V_{\text {MAIN }}$ abruptly increases at the critical current of $I_{\mathrm{cr}}=28.6 \mu \mathrm{A}$, which is independent of the current polarity and the voltage probe pair. The critical Hall electric field $E_{\text {cr }}=6.15 \mathrm{kV} / \mathrm{m}$ calculated from $I_{\text {cr }}$ is consistent with the previous measurements for the intrinsic breakdown of the QHE $13,14,15$

A small DC electric current $I_{\mathrm{MHB}}$ is used to draw a part of hot electrons from the main channel into the MHB. The differential longitudinal resistance $d V_{\mathrm{MHB}} / d I_{\mathrm{MHB}}$ in the MHB is measured with an AC modulation current of $0.05 \mu \mathrm{A}$ and $26.6 \mathrm{~Hz}$ added to $I_{\mathrm{MHB}}$. In Fig. 3, the results with $I_{\mathrm{MHB}}=0.2 \mu \mathrm{A}$ for different voltage probe pairs are shown as a function of $I_{\text {MAIN. }}$. The critical current $I_{\mathrm{cr}}=28.6 \mu \mathrm{A}$ is indicated by arrows. The differential longitudinal resistance in the lower MHB (see Fig. 3(c) and $(\mathrm{d})$ ) steeply increases with $I_{\text {MAIN }}$ for $I_{\text {MAIN }}>I_{\mathrm{cr}}$, while the increase is small in the upper MHB in the region of $I_{\text {MAIN }}>0$ (see Fig. 3(a) and (b)). This indicates that $T_{e}$ is higher in the vicinity of the lower edge than in that of the upper edge. The electric potential due to the Hall effect is higher at the upper edge for $I_{\text {MAIN }}>0$ and the magnetic field direction used here. The results demonstrate that $T_{e}$ increases in the direction of the Hall electric field. In the case of $I_{\text {MAIN }}<0$, the Hall electric field is reversed and a steep increase in $d V_{\mathrm{MHB}} / d I_{\mathrm{MHB}}$ for $I_{\text {MAIN }}<-I_{\text {cr }}$ is observed in the upper MHB. The reversal of the magnetic field also changes the polarity as shown in Fig. 4(a). Similar asymmetric dependence of $d V_{\mathrm{MHB}} / d I_{\mathrm{MHB}}$ on $I_{\mathrm{MAIN}}$ at the center of the $\mathrm{QHE}$ plateau is observed at a lower $T_{\mathrm{L}}$ of $1.5 \mathrm{~K}$, at a higher $N_{s}$ of $2.6 \times 10^{15} \mathrm{~m}^{-2}$ after a LED illumination, and in samples fabricated on other wafers. Fig. 4(b) shows the results at Landau level filling factors higher or lower than $\nu=2$ at the center of the QHE plateau. Overall behaviors are similar to that at $\nu=2$ while $I_{\mathrm{cr}}$ is smaller and $d V_{\mathrm{MHB}} / d I_{\mathrm{MHB}}$ for $\left|I_{\mathrm{MAIN}}\right|<I_{\mathrm{cr}}$ is larger.

The temperature difference between both edges indicates the presence of the component of the heat flow perpendicular to the electric current. Because of $\rho_{x x}>0$ in

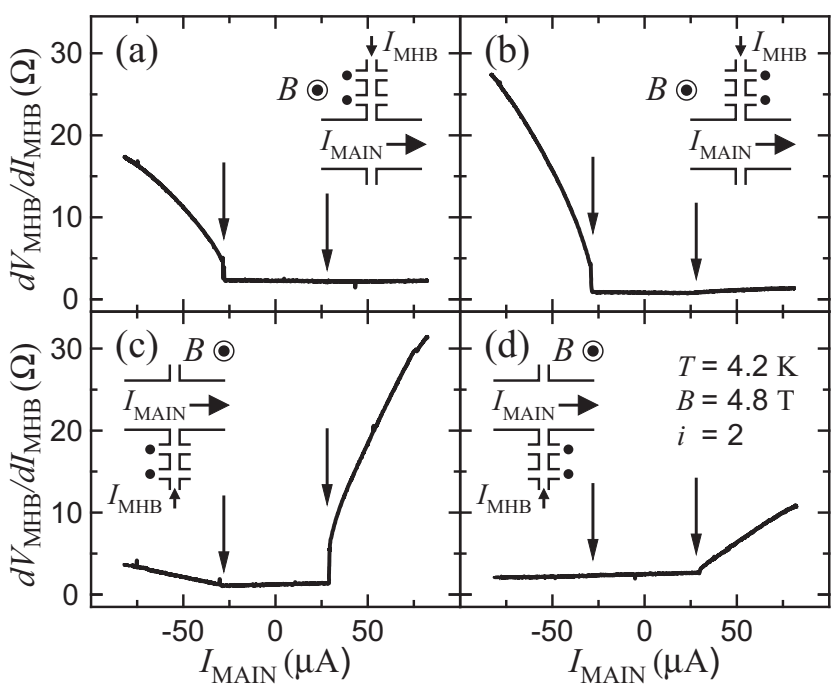

FIG. 3: Differential longitudinal resistance $d V_{\mathrm{MHB}} / d I_{\mathrm{MHB}}$ with $I_{\mathrm{MHB}}=0.2 \mu \mathrm{A}$ in the upper ((a) and (b)) and lower ((c) and $(\mathrm{d}))$ micro Hall bars as a function of electric current $I_{\text {MAIN }}$ through the main channel. The critical current obtained from Fig. 2 is indicated by arrows. The black dots in the insets indicate the voltage probes used in the measurements.

(a)

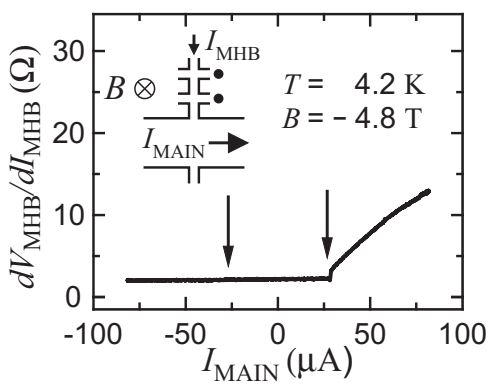

(b)

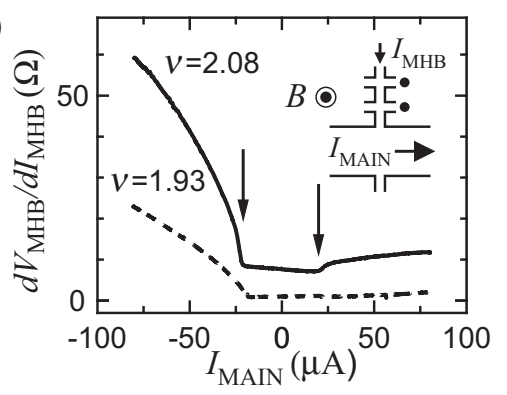

FIG. 4: Differential longitudinal resistance $d V_{\mathrm{MHB}} / d I_{\mathrm{MHB}}$ with $I_{\mathrm{MHB}}=0.2 \mu \mathrm{A}$ in the upper micro Hall bar. (a) Data at the reversed magnetic field. (b) Data at $\nu=1.93$ (dashed curve) with $I_{\mathrm{cr}}=19.9 \mu \mathrm{A}$ and $\nu=2.08$ (solid curve) with $I_{\mathrm{cr}}=21.0 \mu \mathrm{A}$ (indicated by arrows). 
(a)

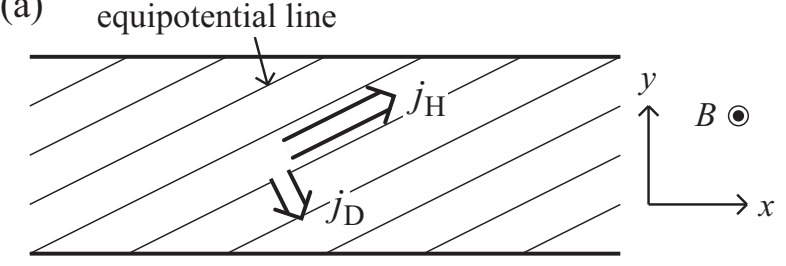

(b)

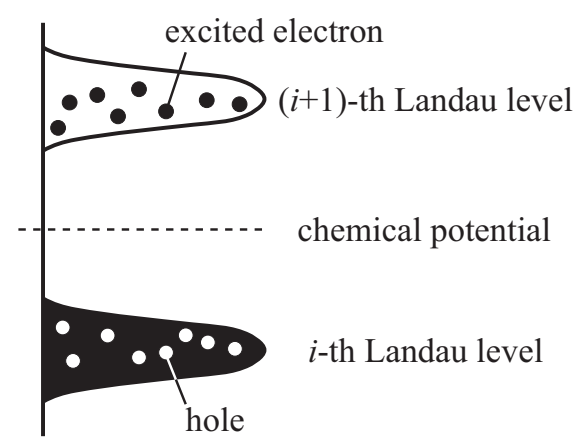

FIG. 5: (a) Schematic view of the Hall current and the dissipative current in a long channel. (b) Excited electrons and holes in the Landau levels. The chemical potential lies at the middle of the gap between the Landau levels at $\nu=$ integer.

the breakdown regime, it is expected that equipotential lines are inclined with respect to the channel direction ( $x$-direction) as shown in Fig. 5(a). The Hall current flows along the equipotential lines and the dissipative current flows across them. In a sufficiently long channel, the $y$-components of the Hall current density $\boldsymbol{j}_{\mathrm{H}}$ and the dissipative current density $\boldsymbol{j}_{\mathrm{D}}$ cancel each other out, i.e. $j_{\mathrm{H}}^{y}+j_{\mathrm{D}}^{y}=0$. On the other hand, the sum of the $y$ components $q_{\mathrm{H}}^{y}$ and $q_{\mathrm{D}}^{y}$ of the heat flow densities $\boldsymbol{q}_{\mathrm{H}}$ and $\boldsymbol{q}_{\mathrm{D}}$ is not zero unless the Peltier coefficient $q_{i} / j_{i}(i=\mathrm{H}$ or $\mathrm{D})$ is the same for both the Hall and dissipative currents. It is expected that the $y$-component of the total heat flow decreases $T_{e}$ around one edge and increases $T_{e}$ around the other.

The experimental results demonstrate that the $y$ component of the heat flow is negative in the case of Fig. $5(\mathrm{a})$. The polarity of the Ettingshausen effect can be explained by Akera's theory ${ }^{11.16}$ Since the direction of the drift motion of electrons is opposite to the Hall current, the $y$-component $q_{\mathrm{H}}^{y}$ of the heat flow density is negative. On the other hand, the dissipative current is carried by excited electrons in the higher Landau level and holes in the lower Landau level (see Fig. 5(b)), which move in opposite directions. At the integer filling factor, the numbers of excited electrons and holes are the same and $q_{\mathrm{D}}^{y}$ cancels out. Thus the total $y$-component of the heat flow density at the center of the quantum Hall plateau is given by $q_{\mathrm{H}}^{y}$, which is negative.

As shown in Fig. 4(b), the polarity of the Ettingshausen effect for $\left|I_{\text {MAIN }}\right|>I_{\text {cr }}$ is the same at $\nu=1.93$ and 2.08 although $q_{\mathrm{D}}^{y}$ is expected to change its sign at $\nu=2.17$ This suggests that $\left|q_{\mathrm{H}} / j_{\mathrm{H}}\right|$ exceeds $\left|q_{\mathrm{D}} / j_{\mathrm{D}}\right|$ in the breakdown regime. 16 The Peltier coefficient $\left|q_{\mathrm{H}} / j_{\mathrm{H}}\right|$ is ex-

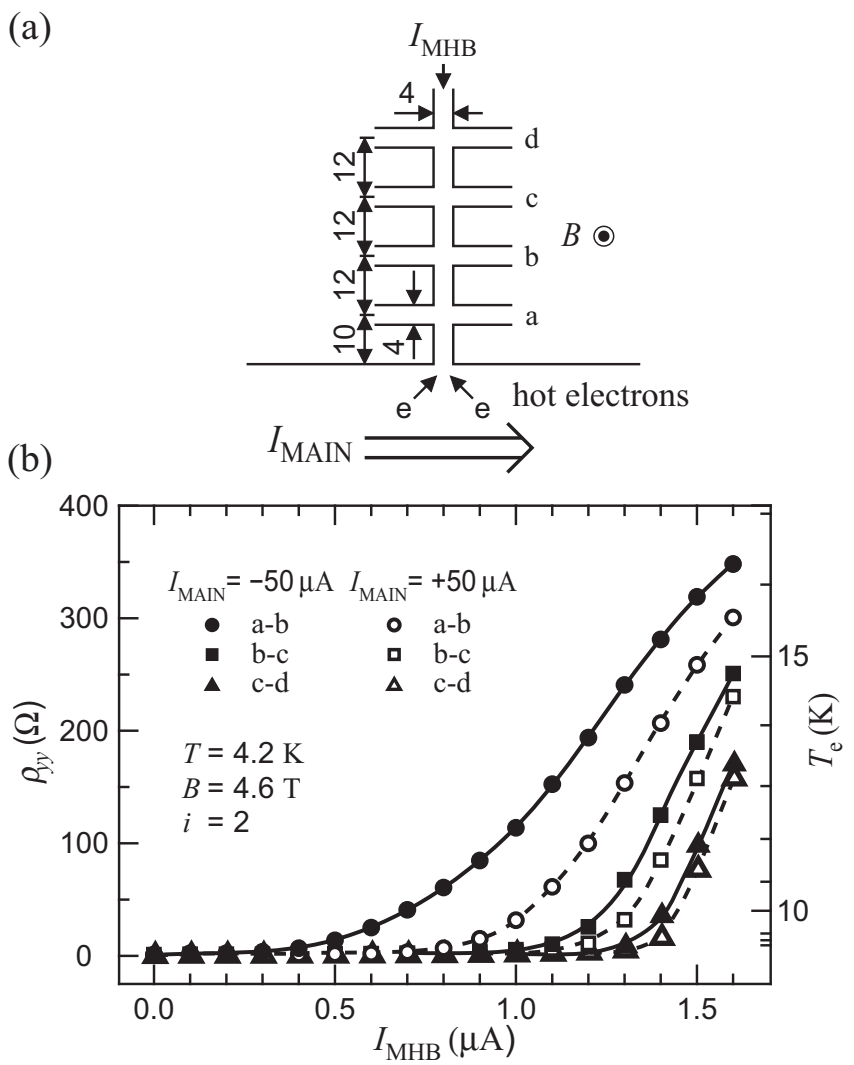

FIG. 6: (a) Geometry of the micro Hall bar for thermal relaxation measurements. The numbers are in units of $\mu \mathrm{m}$. (b) Longitudinal resistivity as a function of the electric current. The full symbols for $I_{\mathrm{MAIN}}=-50 \mu \mathrm{A}$ and the open symbols for $I_{\text {MAIN }}=+50 \mu \mathrm{A}$. Results for various voltage probe pairs are shown. All the lines are guides to the eyes.

pected to be large at high $T_{e}$ since excited electrons and holes carry the heat toward the same direction along the equipotential lines. On the other hand, the creation of pairs of excited electrons and holes does not contribute to $q_{\mathrm{D}}$ while it increases $\left|j_{\mathrm{D}}\right|$. In the region of $\left|I_{\mathrm{MAIN}}\right|<I_{\mathrm{cr}}$, we observed a small linear dependence of $d V_{\mathrm{MHB}} / d I_{\mathrm{MHB}}$ on $I_{\text {MAIN }}$ for Landau level filling factors higher or lower than $\nu=2$. However, the sign of the dependence is negative (positive) for $\nu>2(\nu<2)$ and opposite to that expected from $q_{\mathrm{D}}^{y}$. We consider that it arises from a small change in the Landau level filling factor in the MHB, not from a change in $T_{e}$. The capacitive coupling between the upper and lower edges may lead to a small decrease in the local electron density $(\propto \nu)$ in the upper MHB with increasing Hall voltage $\left(\propto I_{\text {MAIN }}\right)$. This effect produces a change in the longitudinal resistivity which steeply varies with $\nu$ except around the center of the quantum Hall plateau.

In order to investigate the thermal relaxation of hot electrons injected into MHBs, another sample with a MHB having eight voltage probes (Fig. 6(a)) was fabricated on the same wafer, while $N_{s}$ slightly changes. The 
geometry of the other part of the sample is the same as that shown in Fig. 1. Fig. 6(b) shows the longitudinal resistivity $\rho_{y y}$ in the MHB for different voltage probe pairs and different polarities of $I_{\mathrm{MAIN}} \pm 50 \mu \mathrm{A}$. The magnetic field was fixed at the center of the quantum Hall plateau with $i=2$, where $I_{\mathrm{cr}}=26.9 \mu \mathrm{A}$. $T_{e}$ indicated on the right axis is estimated from $T_{\mathrm{L}}$-dependence of the longitudinal resistivity calibrated using a sufficiently small current. The $I_{\mathrm{MAIN}}$-dependence of $\rho_{y y}$ shows that the electron temperature increases with the flux of hot electrons from the main channel. The dependence of $\rho_{y y}$ on the distance from the main channel is negative and associated with the thermal relaxation of hot electrons during transport. In the case of $I_{\mathrm{MAIN}}=0$ or $I_{\mathrm{MHB}}<0$ (not shown in the figure), such dependence on the distance is not observed and the increase in $\rho_{y y}$ is very small except in the region of $\left|I_{\mathrm{MHB}}\right| \gtrsim 1.5 \mu \mathrm{A}$ where the intrinsic (local) breakdown occurs in the MHB. Since $I_{\text {MAIN }}$ is not directly related to the thermal relaxation process in the $\mathrm{MHB}$, the dependence of $\rho_{y y}$ on the polarity of $I_{\mathrm{MAIN}}$ should be attributed to the difference in $T_{e}$ in the vicinity of the upper edge of the main channel due to the Ettingshausen effect discussed above.

Although the origin of the breakdown of the QHE is not clear at the present stage,,$\frac{4}{4}$ the calculation of the critical electric field $E_{\text {cr }}$ based on the heat instability model gives an upper limit of the relaxation time $\tau_{e}$ of hot electrons. Using $E_{\mathrm{cr}}=\left(2 \hbar / m^{*} \tau_{e}\right)^{1 / 2} B$ derived in Ref. 18, $\tau_{e}=2.2 \mathrm{~ns}$ is obtained from the experimental value of
$E_{\mathrm{cr}}=5.79 \mathrm{kV} / \mathrm{m}$. Here, $m^{*}$ is the effective mass of electrons in GaAs (0.067 of the free electron mass). If every hot electron in the MHB propagates with a mean velocity $v_{\mathrm{MHB}}$, the relaxation length is estimated to be only $1.4 \mu \mathrm{m}$ or less for $I_{\mathrm{MHB}}=1 \mu \mathrm{A}\left(v_{\mathrm{MHB}}=695 \mathrm{~m} / \mathrm{s}\right)$. This seems consistent with the fact that, even for the closest voltage probe pair (a-b) with a distance of $10 \mu \mathrm{m}$ from the main channel, $\rho_{y y}$ is much smaller than the longitudinal resistivity $\left(\rho_{x x}=610 \Omega\right)$ in the main channel. The thermal relaxation length $\ell_{\text {edge }}$ that determines the spatial variation of $T_{e}$ in the vicinity of the edges of the main channel can also be estimated from $\tau_{e}$. For example, the $y$-component of the drift velocity along the equipotential lines is calculated to be $109 \mathrm{~m} / \mathrm{s}$ for $\left|I_{\text {MAIN }}\right|=50 \mu \mathrm{A}$. It leads to a very small value of $\ell_{\text {edge }}=0.24 \mu \mathrm{m}$. The change in $T_{e}$ due to the $y$-component of the heat flow is expected to be confined to narrow areas in the vicinity of the edges, while a random potentials or electron-electron interactions might change $\ell_{\text {edge }}$.

In summary, we have observed evidence of the Ettingshausen effect in the breakdown regime of the integer quantum Hall effect. The electron temperature difference between the edges of a long current channel is explained by a heat flow along the equipotential lines.

We would like to thank Professor H. Akera and Dr. Y. Kawano for valuable discussions. This work is supported in part by Grants-in-Aid for Scientific Research from the Ministry of Education, Science, Sports and Culture, Japan.
* Electronic address: komori@dolphin.phys.s.u-tokyo.ac.jp

† Electronic address: okamoto@phys.s.u-tokyo.ac.jp

1 S. Kawaji and J. Wakabayashi, in Physics in High Magnetic Fields, proceedings of the Oji International Seminar, Hakone, Japan, 1980, edited by S. Chikazumi and N. Miura (Springer-Verlag, Berlin, 1981), p. 284.

2 K. von Klitzing, G. Dorda, and M. Pepper, Phys. Rev. Lett. 45, 494 (1980).

3 B. Jeckelmann and B. Jeanneret, Rep. Prog. Phys. 64, 1603 (2001).

4 For a review, see G. Nachtwei, Physica E 4, 79 (1999).

5 S. Komiyama, Y. Kawaguchi, T. Osada, and Y. Shiraki, Phys. Rev. Lett. 77, 558 (1996).

6 I. I. Kaya, G. Nachtwei, K. von Klitzing, and K. Eberl, Phys. Rev. B 58, R7536 (1998).

7 I. I. Kaya, G. Nachtwei, K. von Klitzing, and K. Eberl, Europhys. Lett. 46, 62 (1999).

${ }^{8}$ K. Morita, S. Nomura, H. Tanaka, H. Kawashima, and S. Kawaji, Physica E 12, 169 (2002).

9 For a review, see G. L. Gallagher and P. N. Butcher, in Handbook on Semiconductors Completely Revised Edition edited by T. S. Moss and P. T. Landsberg (Elsevier, Amsterdam, 1992), Vol. 1, Chap. 14, p. 721.

10 For a recent review, see R. Fletcher, Semicond. Sci. Tech- nol. 14, R1 (1999).

11 H. Akera, J. Phys. Soc. Jpn. 71, 228 (2002).

12 In previous works, an asymmetry of the dissipation between the source electrode and the drain electrode has been reported (U. Klaß, W. Dietsche, K. von Klitzing, and K. Ploog, Z. Phys. B 82, 351 (1991); Y. Kawano and S. Komiyama, Phys. Rev. B 68, 085328 (2003)). It is caused by the difference between the entry process of an electron at the source contact and the exit process at the drain contact, and thus not related to the Ettingshausen effect.

13 S. Kawaji, K. Hirakawa, M. Nagata, T. Okamoto, T. Fukase, and T. Gotoh, J. Phys. Soc. Jpn. 63, 2303 (1994).

14 T. Okuno, S. Kawaji, T. Ohrui, T. Okamoto, Y. Kurata, and J. Sakai, J. Phys. Soc. Jpn. 64, 1881 (1995).

15 S. Kawaji, Semicond. Sci. Technol. 11, 1546 (1996).

${ }^{16} \mathrm{H}$. Akera, private communication.

17 The sign of $q_{\mathrm{D}}^{y}$ is expected to be positive (negative) when the chemical potential lies above (below) the middle of the gap between the Landau levels and excited electrons (holes) are the majority carriers (H. Akera and H. Suzuura, unpublished).

18 S. Komiyama and Y. Kawaguchi, Phys. Rev. B 61, 2014 (1999). 